\title{
Measuring End-to-End Quality of a News-on-Demand Web Service
}

\author{
J.R. Arias, F.J. Suárez, D.F. García, and J.I. Marín \\ Departamento de Informática, Universidad de Oviedo, Spain \\ \{arias,fran, daniel, nacho\}@atc.uniovi.es
}

\begin{abstract}
A key issue in any system for distribution of continuous media-on-demand is the capacity of the system with regard to quality of the service specifications, that is, the number of simultaneous streams the system can provide until degradation of quality of reproduction and interactivity perceived by the users. This work presents the evaluation of a web based interactive video-on-demand system, with special attention to limitations caused by the video server. The evaluation is based on the measurement of the end-to-end quality of the service. A set of metrics has been designed to determine the number of streams the video server can support under specific quality requirements. To validate the utility of these metrics, a prototype of a news-on-demand web service has been built and the load for this kind of systems has been characterised. In addition, a load generator which emulates the concurrent access of several users to the system has been built. The evaluation establishes the relationship between the video server limitations and the quality of the service perceived by the users.
\end{abstract}

\section{Introduction}

Examples of commercial video-on-demand services are movies-on-demand and news-on-demand, where the user can interact with the streams through the typical fast-forward, rewind, pause and stop functions. There are two main factors that define the capacity of this kind of system: the service capacity or number of simultaneous streams the system can serve, and the quality of the service perceived by users, both at reproduction of streams and interactivity levels. The greater the number of streams the system can serve, and the higher the quality of reproduction and short response times to interactive functions, the more economically feasible it becomes. The principle elements of the system that limit its capacity are the video server and the communication network. In this work, the influence of the video server, which provides news-on-demand service, is studied, considering the communication network to be ideal. The work focuses on the end-to-end quality of the service, that is, the quality perceived by users in each interaction, from the initial request to the end of the reception of the corresponding stream. The main goal is to design a set of metrics to measure and relate the video server capacity and the end-to-end quality of the service. These metrics will help in dimensioning server capacity in function of the required quality of the service. 


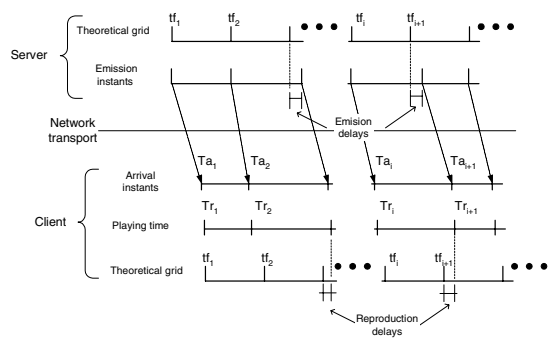

Fig. 1. Multimedia stream reproduction

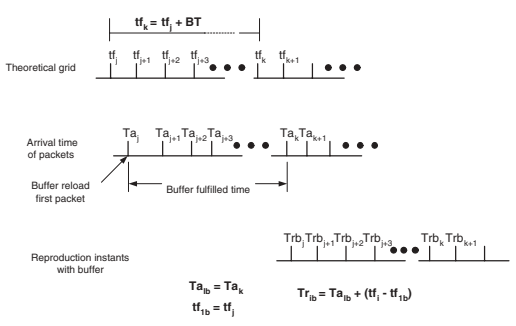

Fig. 2. Stream reproduction with buffer

\section{Prototype of Service}

News-on-demand is one of the most popular on-demand services. To built a prototype representative of real systems the BBC Audio/Video News has been selected as a reference. The synthetic prototype implemented emulates a daily service to which 50 news items are incorporated each day. The prototype contains news for one week. The news items are classified according to their length: a) Short (30 sec.-1 min., $6 \%$ of total), b) Medium (1min. $-3: 30$ min., $84 \%$ of total) or c) Long (3:30 min.-1 hour, 10\% of total). News is available at three transmission speeds: a) Low (28 kbps. maximum), b) Medium (28-56 kbps.) and c) High (more than 56 kbps.). Frame size is always $176 \times 144$, so transmission speed relates directly to frame speed (8-30 fps). For all the news, the video server stores streams coded for the three possible transmission speeds.

\section{Quality of the Service Metrics}

The metrics used to measure quality of the service are based on two fields carried with each packet of the stream transmitted: the sequence number and the timestamp. The sequence number marks the order in which the packet was emitted from the video server. The timestamp places each packet in its temporal position in the stream. These positions form the temporal theoretical grid of emission or playing of the stream. The more the emission/playing of the stream conforms to the theoretical temporal grid, the better the quality of the service obtained. The temporal position of the ith packet in the stream is denoted with $t f_{i}$ and is calculated using the timestamp of each packet and the stream sampling frequency.

\subsection{Metrics of Quality of Reproduction}

Using the value of $t f_{i}$ of the ith packet, the arrival time, $T a_{i}$, of this packet, and the calculated playing time of the ith packet(see Fig. 1), $T r_{i}=T a_{1}+\left(t f_{i}-t f_{1}\right)$, several metrics of quality of reproduction of the stream can be defined.

Percentage of delayed packets: This shows how well the stream received conforms to the theoretical temporal grid. For each packet the delay is calculated 
with $D_{i}=T r_{i}-T a_{i}$. When $D_{i}<0$ there are discontinuities in the stream. If $N_{D}$ is the number of packets delayed, it is possible to calculate the total percentage of delayed packets, $D$, using the total number of packets received, $N_{r}$ as $D=\frac{N_{D}}{N_{r}} * 100$

Mean packet delay: This is the arithmetic mean of the delays: $\bar{R}=\frac{\sum D_{i}}{N_{r}}$ Percentage of packets lost: If $N_{e}$ is the number of packets emitted, this metric is obtained with $L=\frac{N_{e}-N_{r}}{N_{e}} * 100$

Total amount of bytes received: If $L_{i j}$ is the number of bytes carried in the $\mathrm{i}$ th packet received by the $\mathrm{j}$ th client, we can calculate the total amount of bytes received by the clients as $L=\sum_{j=1}^{n} \sum_{i=1}^{m} L_{i j}$, where $n$ is the number of clients connected and $m$ the number of packets received by each client.

Number of client buffer reloads: The client buffer tries to compensate for the transmission delays suffered by the packets. When delays are too long, the packet to play can not be in the buffer. In this case, there is a buffer failure and it must be reloaded completely. The greater the number of reloads, the worse the quality of reproduction of the stream. When the client has a buffer, the new playing time of each packet is $T r_{i b}=T a_{l b}+\left(t f_{i}-t f_{1 b}\right)$, where $T a_{l b}$ is the arrival time of the packet which fills the buffer in the last complete reload, and $t f_{1 b}$ is the temporal position of the first packet to arrive at the buffer in the last reload (see Fig. 22). To determine when a buffer failure is taking place, the arrival time of each packet must be checked: $b f_{i}=T r_{i b}-T a_{i}$. When $b f_{i}<0$ there is a buffer failure and the buffer must be reloaded. The total number of a client buffer failures is calculated as $B F=\sum b f_{i}$.

Buffer reloads frecuency: If $T$ is the time spent by a client requesting multimedia streams to the server, the metric value is $F R=\frac{B F}{T}$.

Buffer Reload Mean Time: When a buffer failure is detected a reload must be started. If the time spended by the client awaiting for the complete fullfil of the buffer is too long, clients will be leads to abandon the service. We measure this parameter with this metric.

\subsection{Metrics of Quality of Interactivity}

Mean start response time: quantifies waiting time between a request for the start of a stream and confirmation of the transmission from the server.

Mean interaction response time: measures the waiting time between client interaction and response from the server.

\subsection{Metrics of Server Capacity}

These metrics relate server load with the number of client requests.

\begin{tabular}{ll}
\hline Throughput metrics & Resource utilization metrics \\
\hline \hline Streams served & CPU utilization \\
Total number of bits served & Hard disk utilization \\
Mean number of concurrent connections & Network utilization \\
Total number of bits served per connection & \\
\hline
\end{tabular}




\section{Workload Characterization and Experimental Procedure}

In the service model proposed in this paper the user can request as much news as he wants. The choice of news is made randomly among all available news. The access probability to each piece of news is driven by the Zipf law 3. According to [2], half of the requests are abandoned before completion due to lack of interest on the part of the user. Each one of the client states and the thinking time before the selection of a new stream has a length which follows constant statistical distributions. These distributions are based on the work presented in [2].

The experimental procedure was carried out with two computers, one of them working as the news server and the other playing the role of multiple clients by running a specific emulation tool. The computers were linked by a dedicated 100 $\mathrm{Mb} / \mathrm{s}$ Fast-Ethernet. Measurements were taken in both computers: in the server using the sar system utility and in the client using the specific tool built.

\section{Current Results}

A preliminary analysis of the prototype, without considering the use of buffers in the clients, has been carried out. The analysis is based on the value of the metrics as a function of the number of users simultaneously connected to the server. As a result, can be concluded that the server application (Darwin [1] server in this work) does not go beyond a certain level of throughput, even if the hardware resources do not reach saturation levels. Just before reaching the maximum throughput, as the throughput (load level) of the server increases, the server application discards more and more packets with the goal of maintaining the average delay of packets at a minimum. This behaviour of server application is reflected in the metrics as a progressive increase in lost packets, while the average delay of packets remains stationary with low values. Once the point of maximum throughput has been reached, the loss of packets stabilizes.

In the experimental framework of this work, the prototype of the news-ondemand service used has capacity for: a) 120 users for high speed, b) 200 users for medium speed, and c) 260 users for low speed, while maintaining an acceptable quality of the service. With these numbers of supported users using economical personal computers as servers, the economic feasibility of interactive video-ondemand services is evident.

\section{References}

1. Apple Computer Inc. Open source streaming server, 2002. http://www.publicsource.apple.com/projects/streaming/.

2. S. Jin and A. Bestavros. A generator of internet streaming media objects and workloads. Technical report, Boston University. Computer Science Department, October 2001. http://citeseer.nj.nec.com/jin01generator.html.

3. G.K. Zipf. The human behaviour and the principle of least effort. Addison Wesley, 1949. 